Bulletin of the Section of Logic

Volume 45/2 (2016), pp. 79-91

http://dx.doi.org/10.18778/0138-0680.45.2.02

Mitio Takano

\title{
AXIOMATIZATION OF A BASIC LOGIC OF LOGICAL BILATTICES
}

\begin{abstract}
A sequential axiomatization is given for the 16-valued logic that has been proposed by Shramko-Wansing (J Philos Logic 34:121-153, 2005) as a candidate for the basic logic of logical bilattices.

Keywords: logical bilattice, sequent calculus

\section{Introduction}

As a candidate for the basic logic of logical bilattices, Shramko-Wansing [5] proposed the 16-valued logic that originates from the bilattice $S I X T E E N_{2}$, instead of the logic of Arieli-Avron [1] originating from the bilattice $F O U R_{2}$. The latter has the elegant cut-free sequent calculus $G B L$ in [1].

The main result of this paper is to give a sound and complete sequent calculus for the former logic. But regrettably, cut plays the central role in our calculus, and so refinements are expected. By the way, it is noted that Shramko-Wansing's logic can be considered to be the expansion of ArieliAvron's one by the connective $\sim_{f}$ for $f$-inversion (cf. Subsection 4.1).

The language without propositional constants is considered first, and the one with them is mentioned in the last section. As with [6], this work was highly motivated by Odintsov [3].

For logical bilattices, see Arieli-Avron [1].
\end{abstract}




\section{Validity and provability}

The unary connective $\sim_{b}$ is introduced to represent the concatenation $\sim_{f} \sim_{t}$ for brevity's sake. So, formulas are constructed from propositional variables by means of the unary connectives $\sim_{t}, \sim_{f}, \sim_{b}$ and the binary connectives $\wedge_{t}, \vee_{t}, \wedge_{f}, \vee_{f}$. Propositional variables, formulas and finite sets of formulas are denoted by $p, q, \ldots, A, B, \ldots$ and $\Gamma, \Delta, \ldots$, respectively.

As the representation of semantics of the logics, the one presented in Odintsov [3] is adopted as with [6].

Definition 2.1 (Shramko-Wansing [5], Odintsov [3]). (1) A valuation is a function from the set of the propositional variables into the set $\{1,0\}^{4}$ of the quadruplets consisting of the classical truth values 1 (truth) and 0 (falsity).

(2) A valuation $v$ is extended uniquely to the set of the formulas by the following recursion, where $v(A)=(n, f, t, b), v(B)=\left(n^{\prime}, f^{\prime}, t^{\prime}, b^{\prime}\right)$, and $\wedge$ (respectively, $\vee$ ) denotes the conjunction (respectively, disjunction) operator on 1 and 0 :

$$
\begin{aligned}
v\left(\sim_{t} A\right) & =(t, b, n, f) ; \\
v\left(\sim_{f} A\right) & =(f, n, b, t) ; \\
v\left(\sim_{b} A\right) & =(b, t, f, n) ; \\
v\left(A \wedge_{t} B\right) & =\left(n \vee n^{\prime}, f \vee f^{\prime}, t \wedge t^{\prime}, b \wedge b^{\prime}\right) ; \\
v\left(A \vee_{t} B\right) & =\left(n \wedge n^{\prime}, f \wedge f^{\prime}, t \vee t^{\prime}, b \vee b^{\prime}\right) ; \\
v\left(A \wedge_{f} B\right) & =\left(n \wedge n^{\prime}, f \vee f^{\prime}, t \wedge t^{\prime}, b \vee b^{\prime}\right) ; \\
v\left(A \vee_{f} B\right) & =\left(n \vee n^{\prime}, f \wedge f^{\prime}, t \vee t^{\prime}, b \wedge b^{\prime}\right) .
\end{aligned}
$$

When $v$ is a valuation and $v(A)=(n, f, t, b)$, the classical truth values $n, f, t$ and $b$ are denoted by $v_{n}(A), v_{f}(A), v_{t}(A)$ and $v_{b}(A)$, respectively.

According to Shramko-Wansing [5, DEFINITION 6.7], the designated truth values are $\boldsymbol{T}, \mathbf{N T}, \mathbf{T B}$ and $\mathbf{N T B}$, and these correspond to our quadruple truth values $(0,0,1,0),(1,0,1,0),(0,0,1,1)$ and $(1,0,1,1)$, respectively. So, the following definition of the set des of the designated truth values is posed:

$$
\text { des }=\{(n, 0,1, b) \mid n, b \in\{1,0\}\} .
$$


Definition 2.2 (Shramko-Wansing [5]). The entailment relation $\models_{b l}$ is defined as follows: $A \models_{b l} B$, iff for every valuation $v$, if $v(A) \in$ des then $v(B) \in$ des.

A sequent is an expression of the form $\Gamma \Rightarrow \Delta$, while $\Gamma$ and $\Delta$ are its antecedent and succedent respectively. The expression $A \Longleftrightarrow B$ is the abbreviation for the two sequents $A \Rightarrow B$ and $B \Rightarrow A$.

Definition 2.3. A sequent $\Gamma \Rightarrow \Delta$ is bl-valid, iff for every valuation $v$, either $v(A) \notin$ des for some $A \in \Gamma$, or $v(B) \in$ des for some $B \in \Delta$.

Clearly, $A \models_{b l} B$ iff the sequent $A \Rightarrow B$ is $b l$-valid. It is to be noted that the sequent $A \vee_{t} B \Rightarrow A, B$ is not necessarily $b l$-valid, since it is possible that $v_{f}(A)=v_{t}(A)=1$ and $v_{f}(B)=v_{t}(B)=0$ for some $A, B$ and some valuation $v$; this causes the difficulty of axiomatizing $b l$-validity.

Before presenting our system, the formulas $X_{i}$ and $Y_{i}(i=1,2, \ldots, 21)$ are defined by the following table:

\begin{tabular}{ccc|ccc}
\hline$i$ & $X_{i}$ & $Y_{i}$ & $i$ & $X_{i}$ & $Y_{i}$ \\
\hline 1 & $\sim_{t} \sim_{t} A$ & $A$ & 12 & $\sim_{t}\left(A \wedge_{f} B\right)$ & $\sim_{t} A \wedge_{f} \sim_{t} B$ \\
2 & $\sim_{t} \sim_{f} A$ & $\sim_{b} A$ & 13 & $\sim_{t}\left(A \vee_{f} B\right)$ & $\sim_{t} A \vee_{f} \sim_{t} B$ \\
3 & $\sim_{t} \sim_{b} A$ & $\sim_{f} A$ & 14 & $\sim_{f}\left(A \wedge_{t} B\right)$ & $\sim_{f} A \wedge_{t} \sim_{f} B$ \\
4 & $\sim_{f} \sim_{t} A$ & $\sim_{b} A$ & 15 & $\sim_{f}\left(A \vee_{t} B\right)$ & $\sim_{f} A \vee_{t} \sim_{f} B$ \\
5 & $\sim_{f} \sim_{f} A$ & $A$ & 16 & $\sim_{f}\left(A \wedge_{f} B\right)$ & $\sim_{f} A \vee_{f} \sim_{f} B$ \\
6 & $\sim_{f} \sim_{b} A$ & $\sim_{t} A$ & 17 & $\sim_{f}\left(A \vee_{f} B\right)$ & $\sim_{f} A \wedge_{f} \sim_{f} B$ \\
7 & $\sim_{b} \sim_{t} A$ & $\sim_{f} A$ & 18 & $\sim_{b}\left(A \wedge_{t} B\right)$ & $\sim_{b} A \vee_{t} \sim_{b} B$ \\
8 & $\sim_{b} \sim_{f} A$ & $\sim_{t} A$ & 19 & $\sim_{b}\left(A \vee_{t} B\right)$ & $\sim_{b} A \wedge_{t} \sim_{b} B$ \\
9 & $\sim_{b} \sim_{b} A$ & $A$ & 20 & $\sim_{b}\left(A \wedge_{f} B\right)$ & $\sim_{b} A \vee_{f} \sim_{b} B$ \\
10 & $\sim_{t}\left(A \wedge_{t} B\right)$ & $\sim_{t} A \vee_{t} \sim_{t} B$ & 21 & $\sim_{b}\left(A \vee_{f} B\right)$ & $\sim_{b} A \wedge_{f} \sim_{b} B$ \\
11 & $\sim_{t}\left(A \vee_{t} B\right)$ & $\sim_{t} A \wedge_{t} \sim_{t} B$ & & & \\
\hline
\end{tabular}

It is easy to see $v\left(X_{i}\right)=v\left(Y_{i}\right)$ for every valuation $v(i=1,2, \ldots, 21)$ (Takano [6, Lemma 3.2]).

Definition 2.4. Our system $\mathrm{L}_{b l}$ consists of the axioms and the inference rules.

First, the axioms are as follows.

(A1) $A \Rightarrow A \vee_{t} B$

(A2) $A \vee_{t} B \Rightarrow B \vee_{t} A$ 
(A3) $\left(A \vee_{t} B\right) \vee_{t} C, C \vee_{t} D \Rightarrow D, A \vee_{t} C, B \vee_{t} C$

(A4) $A \vee_{t} C \Rightarrow\left(A \vee_{t} B\right) \vee_{t} C$ and $B \vee_{t} C \Rightarrow\left(A \vee_{t} B\right) \vee_{t} C$

(A5) $\left(A \wedge_{t} B\right) \vee_{t} C \Rightarrow A \vee_{t} C$ and $\left(A \wedge_{t} B\right) \vee_{t} C \Rightarrow B \vee_{t} C$

(A6) $A \vee_{t} C, B \vee_{t} C \Rightarrow\left(A \wedge_{t} B\right) \vee_{t} C$

(A7) $\sim_{b} A, A \Rightarrow$

(A8) $\sim_{b} A \vee_{t} C, A \Rightarrow C$

(A9) $A \vee_{t} C \Rightarrow A, \sim_{b} A \vee_{t} C$

(B1) $\left(A \wedge_{f} B\right) \vee_{t} C \Longleftrightarrow\left(A \wedge_{t} B\right) \vee_{t} C$

(B2) $\left(A \vee_{f} B\right) \vee_{t} C \Longleftrightarrow\left(A \vee_{t} B\right) \vee_{t} C$

(Ci) $X_{i} \vee_{t} C \Longleftrightarrow Y_{i} \vee_{t} C \quad(i=1,2, \ldots, 21)$

The inference rules, on the other hand, are as follows.

$$
\text { (Extension) } \frac{\Gamma \Rightarrow \Delta}{\Gamma, \Pi \Rightarrow \Delta, \Lambda} \quad \text { (Cut) } \frac{\Gamma \Rightarrow \Delta, A \quad A, \Pi \Rightarrow \Lambda}{\Gamma, \Pi \Rightarrow \Delta, \Lambda}
$$

Provability of a sequent (in $\mathrm{L}_{b l}$ ) is defined in the standard way.

Remark 2.5. The sequent $\sim_{b} A, A \Rightarrow C$ is obtained from Axioms (A1) and (A8) by an application of (Cut). But, now that sequents with the empty succedents are allowed, Axiom (A7) (or another axiom with the empty succedent) is indispensable in $\mathrm{L}_{b l}$, since every provable sequent has the non-empty succedent without it (cf. Remark $5.2(2)$ ).

Now, the main result of this paper is formulated as the following theorem.

THEOREM 2.6. A sequent is bl-valid iff it is provable in $\mathrm{L}_{b l}$. In particular, $A \models_{b l} B$ iff the sequent $A \Rightarrow B$ is provable.

It is routine to check the 'if' part of the theorem (soundness), and proof of the 'only if' part (completeness) is given in the next section.

Lemma 2.7. Suppose $\Gamma \neq \emptyset$. If $\Gamma \vee_{t} C \Rightarrow B \vee_{t} C$ is provable for every $C$, then $\Gamma \Rightarrow B$ is provable, where $\Gamma \vee_{t} C=\left\{A \vee_{t} C \mid A \in \Gamma\right\}$.

Proof: Suppose $\Gamma=\left\{A_{0}, A_{1}\right\}$, for simplicity. Then, the conclusion follows from the following derivation: 
1.

2 .

3.

4.

5.

6.

7.

8.

9.

$$
\begin{aligned}
A_{0} & \Rightarrow A_{0} \vee_{t} \sim_{b} A_{0} \\
A_{1} & \Rightarrow A_{1} \vee_{t} \sim_{b} A_{0} \\
A_{0} \vee_{t} \sim_{b} A_{0}, A_{1} \vee_{t} \sim_{b} A_{0} & \Rightarrow B \vee_{t} \sim_{b} A_{0} \\
A_{0}, A_{1} \vee_{t} \sim_{b} A_{0} & \Rightarrow B \vee_{t} \sim_{b} A_{0} \\
A_{0}, A_{1} & \Rightarrow B \vee_{t} \sim_{b} A_{0} \\
B \vee_{t} \sim_{b} A_{0} & \Rightarrow \sim_{b} A_{0} \vee_{t} B \\
A_{0}, A_{1} & \Rightarrow \sim_{b} A_{0} \vee_{t} B \\
\sim_{b} A_{0} \vee_{t} B, A_{0} & \Rightarrow B \\
A_{0}, A_{1} & \Rightarrow B
\end{aligned}
$$

assumption

Note that the Grundsequenz $A \Rightarrow A$ is out of use in the above derivation (cf. Lemma 2.9).

The following is an immediate corollary by Axioms (A5), (A6), (B1), (B2) and $(\mathrm{C} i)$, respectively.

Corollary 2.8. The following sequents are provable.

(1) $A \wedge_{t} B \Rightarrow A$ and $A \wedge_{t} B \Rightarrow B$

(2) $A, B \Rightarrow A \wedge_{t} B$

(3) $A \wedge_{f} B \Longleftrightarrow A \wedge_{t} B$

(4) $A \vee_{f} B \Longleftrightarrow A \vee_{t} B$

$(5)_{i} \quad X_{i} \Longleftrightarrow Y_{i} \quad(i=1,2, \ldots, 21)$

Lemma 2.9. The sequent $A \Rightarrow A$ is provable.

Proof: $A \Rightarrow A$ follows from $A \Rightarrow A \wedge_{t} A$ and $A \wedge_{t} A \Rightarrow A$, which are instances of Corollary 2.8 (2) and (1) respectively, by (Cut).

\section{Completeness}

The proof of the 'only if' part of Theorem 2.6 (completeness) is given in this section. 
Definition 3.1 (Maehara [2]). A set $\alpha$ of formulas forms a complete consistent system, iff the sequent $\Pi \Rightarrow \Lambda$ is unprovable for every finite sets $\Pi$ and $\Lambda$ of formulas such that $\Pi \subseteq \alpha$ and $\Lambda \cap \alpha=\emptyset$.

Lemma 3.2 (Maehara [2, Theorem 1]). If a sequent $\Gamma \Rightarrow \Delta$ is unprovable, then there is a complete consistent system $\alpha$ such that $\Gamma \subseteq \alpha$ and $\Delta \cap \alpha=\emptyset$.

Proof: Beginning with $\Gamma \Rightarrow \Delta$, add each formula one-by-one to the antecedent or succedent while keeping its unprovability. Then, the set of all formulas in the antecedents forms a desired one.

Then, the 'only if' part of Theorem 2.6, namely, validity implies provability, immediately follows from Lemmas 3.2 and 3.6 below. For, given an unprovable sequent $\Gamma \Rightarrow \Delta$, take a complete consistent system $\alpha$ such that $\Gamma \subseteq \alpha$ and $\Delta \cap \alpha=\emptyset$ by Lemma 3.2, then under the valuation $v^{0}$ defined in Definition 3.4, $A \in \Gamma$ implies $v^{0}(A) \in$ des by Lemma 3.6 since $A \in \alpha$, while $B \in \Delta$ implies $v^{0}(B) \notin$ des by Lemma 3.6 again since $B \notin \alpha$, and so $\Gamma \Rightarrow \Delta$ is not $b l$-valid.

So, let $\alpha$ be a complete consistent system, and be fixed in the rest of this section. Moreover, if it is the case that

$$
A \vee_{t} B \in \alpha \text { implies } A \in \alpha \text { or } B \in \alpha \text {, for every } A \text { and } B,
$$

then let $F(p)$ be $p$ itself. If otherwise, fix a pair $C, D$ such that $C \vee_{t} D \in \alpha$ but $C, D \notin \alpha$, and let $F(p)$ be $p \vee_{t} C$. So, $F\left(A \wedge_{t} B\right)$ denotes $A \wedge_{t} B$ or $\left(A \wedge_{t} B\right) \vee_{t} C$ accordingly, for example.

LEMMA 3.3.

(1) $\quad F\left(A \wedge_{t} B\right) \in \alpha$, iff $F(A) \in \alpha$ and $F(B) \in \alpha$.

(2) $F\left(A \vee_{t} B\right) \in \alpha$, iff $F(A) \in \alpha$ or $F(B) \in \alpha$.

(3) $F\left(A \wedge_{f} B\right) \in \alpha$, iff $F(A) \in \alpha$ and $F(B) \in \alpha$.

(4) $F\left(A \vee_{f} B\right) \in \alpha$, iff $F(A) \in \alpha$ or $F(B) \in \alpha$.

$(5)_{i} \quad F\left(X_{i}\right) \in \alpha$, iff $F\left(Y_{i}\right) \in \alpha . \quad(i=1,2, \ldots, 21)$

(6) $A \in \alpha$, iff $F(A) \in \alpha$ but $F\left(\sim_{b} A\right) \notin \alpha$.

Proof: Recall that, since $\alpha$ forms a complete consistent system, if the sequent $\Pi \Rightarrow \Lambda$ is provable, then either $\Pi \nsubseteq \alpha$ or $\Lambda \cap \alpha \neq \emptyset$.

Case 1. $\left(^{*}\right)$ is the case, and so $F(p)$ is $p:$ (1) By Corollary 2.8 (1) and (2). (2) By the assumption (*) and Axioms (A1), (A2). (3) By (1) 
and Corollary 2.8 (3). (4) By (2) and Corollary 2.8 (4). (5) $i$ By Corollary $2.8(5)_{i}$. (6) By Axiom (A7).

Case 2. (*) is not the case, and so $C \vee_{t} D \in \alpha$ but $C, D \notin \alpha$, and $F(p)$ is $p \vee_{t} C$ : (1) By Axioms (A5) and (A6). (2) The 'only if' part follows from Axiom (A3) and the assumption that $C \vee_{t} D \in \alpha$ but $D \notin \alpha$. While the 'if' part follows from Axiom (A4). (3) By (1) and Axiom (B1). (4) By (2) and Axiom (B2). (5) $i$ By Axiom $(\mathrm{C} i$ ). (6) The 'only if' part follows from Axioms (A1), (A8) and the assumption $C \notin \alpha$. While the 'if' part follows from Axiom (A9).

DEFINITION 3.4. Let $v^{0}$ be the valuation such that for every propositional variable $p$ :

$$
\begin{array}{llll}
v_{n}^{0}(p)=1 \quad \text { iff } F\left(\sim_{t} p\right) \in \alpha ; & v_{f}^{0}(p)=1 \quad \text { iff } F\left(\sim_{b} p\right) \in \alpha ; \\
v_{t}^{0}(p)=1 \quad \text { iff } F(p) \in \alpha ; & v_{b}^{0}(p)=1 \quad \text { iff } F\left(\sim_{f} p\right) \in \alpha .
\end{array}
$$

Lemma 3.5. The following properties hold for every formula $E$.

$$
\begin{array}{lll}
v_{n}^{0}(E)=1 \quad \text { iff } F\left(\sim_{t} E\right) \in \alpha ; & v_{f}^{0}(E)=1 \quad \text { iff } F\left(\sim_{b} E\right) \in \alpha ; \\
v_{t}^{0}(E)=1 \quad \text { iff } F(E) \in \alpha ; & v_{b}^{0}(E)=1 \quad \text { iff } F\left(\sim_{f} E\right) \in \alpha .
\end{array}
$$

Proof: By simultaneous induction on the construction of $E$.

Case 1. E is a propositional variable: Evident by the definition.

Case 2. $E$ is $\sim_{t} A: v_{t}^{0}\left(\sim_{t} A\right)=1$, iff $v_{n}^{0}(A)=1$, iff $F\left(\sim_{t} A\right) \in \alpha$ by induction hypothesis; $v_{n}^{0}\left(\sim_{t} A\right)=1$, iff $v_{t}^{0}(A)=1$, iff $F(A) \in \alpha$ by induction hypothesis, iff $F\left(\sim_{t} \sim_{t} A\right) \in \alpha$ by Lemma $3.3(5)_{1}$; the remainder are similar.

Cases 3-4. E is $\sim_{f} A$ or $\sim_{b} A$ : Similar to Case 2.

Case 5. $E$ is $A \wedge_{t} B: v_{t}^{0}\left(A \wedge_{t} B\right)=1$, iff $v_{t}^{0}(A)=1$ and $v_{t}^{0}(B)=1$, iff $F(A) \in \alpha$ and $F(B) \in \alpha$ by induction hypothesis, iff $F\left(A \wedge_{t} B\right) \in \alpha$ by Lemma $3.3(1) ; v_{n}^{0}\left(A \wedge_{t} B\right)=1$, iff $v_{n}^{0}(A)=1$ or $v_{n}^{0}(B)=1$, iff $F\left(\sim_{t} A\right) \in$ $\alpha$ or $F\left(\sim_{t} B\right) \in \alpha$ by induction hypothesis, iff $F\left(\sim_{t} A \vee_{t} \sim_{t} B\right) \in \alpha$ by Lemma 3.3 (2), iff $F\left(\sim_{t}\left(A \wedge_{t} B\right)\right) \in \alpha$ by Lemma $3.3(5)_{10}$; the remainder are similar.

Cases 6-8. $E$ is $A \vee_{t} B, A \wedge_{f} B$ or $A \vee_{f} B$ : Similar to Case 5. 
Lemma 3.6. $A \in \alpha$ iff $v^{0}(A) \in$ des, for every formula $A$.

Proof: $A \in \alpha$, iff $F(A) \in \alpha$ but $F\left(\sim_{b} A\right) \notin \alpha$ by Lemma 3.3 (6), iff $v_{t}^{0}(A)=1$ but $v_{f}^{0}(A)=0$ by Lemma 3.5, iff $v^{0}(A) \in$ des.

\section{Two fragment logics}

Digressing from the main theme, this section is devoted to fragment logics. In this section only, valuations in the sense of Definition 2.1 are called NFTB-valuations, to make a distinction from NT- and FT-valuations introduced below.

\subsection{The $\left\{\sim_{t}, \wedge_{t}, \vee_{t}, \wedge_{f}, \vee_{f}\right\}$-fragment}

Let's ignore the connectives $\sim_{f}$ and $\sim_{b}$, as well as the second and fourth components of the quadruple truth values. Then, an NFTB-valuation becomes a function $w$, NT-valuation, from the set of the propositional variables into the set $\{1,0\}^{2}$, and is extended to the set of the formulas in this fragment by the following recursion, where $w(A)=(n, t)$ and $w(B)=\left(n^{\prime}, t^{\prime}\right)$ :

$$
\begin{aligned}
w\left(\sim_{t} A\right) & =(t, n) ; \\
w\left(A \wedge_{t} B\right) & =\left(n \vee n^{\prime}, t \wedge t^{\prime}\right) ; \\
w\left(A \vee_{t} B\right) & =\left(n \wedge n^{\prime}, t \vee t^{\prime}\right) ; \\
w\left(A \wedge_{f} B\right) & =\left(n \wedge n^{\prime}, t \wedge t^{\prime}\right) ; \\
w\left(A \vee_{f} B\right) & =\left(n \vee n^{\prime}, t \vee t^{\prime}\right),
\end{aligned}
$$

while the set des of the designated truth values becomes the set $d e s^{\prime}=$ $\{(1,1),(0,1)\}$, and the definition of validity of a sequent in the fragment becomes as follows: $\Gamma \Rightarrow \Delta$ is valid, NT-valid, iff for every NT-valuation $w$, either $w(A) \notin d e s^{\prime}$ for some $A \in \Gamma$, or $w(B) \in d e s^{\prime}$ for some $B \in \Delta$.

Then, the logic determined by NT-validity is nothing but the basic logic of logical bilattices studied in Arieli-Avron [1], where the connectives $\sim_{t}, \wedge_{t}, \vee_{t}, \wedge_{f}$ and $\vee_{f}$ correspond to their $\neg, \wedge, \vee, \otimes$ and $\oplus$, respectively. For, NT-validity of $\Gamma \Rightarrow \Delta$ is identical with their $\Gamma \models_{\langle F O U R\rangle} \Delta$, and the latter is equivalent to $\Gamma \models_{B L} \Delta$ ([1, THEOREM 3.4]).

Moreover, according to the following lemma, the logic proposed by Shramko-Wansing [5] can be considered to be simply the expansion of the 
one by Arieli-Avron [1] by the introduction of the connective $\sim_{f}$ (and so $\sim_{b}$ ).

Lemma 4.1. For every $A$ and $B$ in the $\left\{\sim_{t}, \wedge_{t}, \vee_{t}, \wedge_{f}, \vee_{f}\right\}$-fragment, the following conditions (BL1)-(BL3) are mutually equivalent.

(BL1) $A=_{b l} B$.

(BL2) The sequent $A \Rightarrow B$ is NT-valid.

(BL3) $v_{t}(A)=1$ implies $v_{t}(B)=1$, for every NFTB-valuation $v$.

It is to be noted that this lemma cannot be extended to the sequents having plural succedent formulas, since $A \vee_{t} B \Rightarrow A, B$ is NT-valid, but is not necessarily $b l$-valid.

For the proof, the following one is convenient.

Lemma 4.2 (Shramko-Wansing [5, LEMMA 4.8]). For every $A$ and $B$ (in the full language), the following two conditions are mutually equivalent.

- $v_{t}(A)=1$ implies $v_{t}(B)=1$, for every NFTB-valuation $v$.

- $v_{f}(B)=1$ implies $v_{f}(A)=1$, for every NFTB-valuation $v$.

Proof OF LeMMA 4.1:

From (BL1) to (BL2): Suppose (BL1). Let $w$ be an arbitrary NT-valuation. It suffices to show that $w(A) \in$ des $^{\prime}$ implies $w(B) \in$ des $^{\prime}$. Let $\bar{w}$ be the NFTB-valuation such that $\bar{w}(p)=\left(w_{n}(p), 0, w_{t}(p), 0\right)$ for every $p$. Then it is easily seen by induction that $\bar{w}(E)=\left(w_{n}(E), 0, w_{t}(E), 0\right)$, and so $w(E) \in d e s^{\prime}$ iff $\bar{w}(E) \in$ des (iff $w_{t}(E)=1$ ), for every $E$ in the fragment. So, if $w(A) \in d e s^{\prime}$, then $\bar{w}(A) \in$ des, so $\bar{w}(B) \in$ des by (BL1), and so $w(B) \in d e s^{\prime}$.

From (BL2) to (BL3): Suppose (BL2). Let $v$ be an arbitrary NFTB-valuation, and $\hat{v}$ be the NT-valuation such that $\hat{v}(p)=\left(v_{n}(p), v_{t}(p)\right)$ for every $p$. Then $\hat{v}(E)=\left(v_{n}(E), v_{t}(E)\right)$, and so $\hat{v}(E) \in d e s^{\prime}$ iff $v_{t}(E)=1$, for every $E$ in the fragment. So, if $v_{t}(A)=1$, then $\hat{v}(A) \in d e s^{\prime}$, so $\hat{v}(B) \in d e s^{\prime}$ by $(\mathrm{BL} 2)$, and so $v_{t}(B)=1$.

From (BL3) to (BL1): Suppose (BL3). Let $v$ be an arbitrary NFTB-valuation. We must show that $v(A) \in$ des implies $v(B) \in$ des. So, suppose $v(A) \in$ des, namely, $v_{t}(A)=1$ and $v_{f}(A)=0$. The former implies $v_{t}(B)=1$ by (BL3), while the latter implies $v_{f}(B)=0$ by (BL3) again together with Lemma 4.2; hence $v(B) \in$ des. 


\subsection{The $\left\{\sim_{b}, \wedge_{t}, \vee_{t}\right\}$-fragment}

Now, ignore the connectives $\sim_{t}, \sim_{f}, \wedge_{f}$ and $\vee_{f}$, as well as the first and fourth components of the quadruple truth values, instead. In this case, a valuation becomes a function $w$, FT-valuation, from the set of the propositional variables into the set $\{1,0\}^{2}$, and is extended to the set of the formulas in this fragment by the following recursion, where $w(A)=(f, t)$ and $w(B)=\left(f^{\prime}, t^{\prime}\right)$ :

$$
\begin{aligned}
w\left(\sim_{b} A\right) & =(t, f) ; \\
w\left(A \wedge_{t} B\right) & =\left(f \vee f^{\prime}, t \wedge t^{\prime}\right) ; \\
w\left(A \vee_{t} B\right) & =\left(f \wedge f^{\prime}, t \vee t^{\prime}\right),
\end{aligned}
$$

while the set des of the designated truth values becomes the set des" $=$ $\{(0,1)\}$, and the modified notion of validity, FT-validity, is determined accordingly. Moreover, the system $\mathrm{L}_{b l}$ becomes the subsystem that consists of the axioms (A1)-(A9), (C9), (C18) and (C19), as well as the inference rules (Extension) and (Cut). Then, by deleting the items concerning the connectives $\sim_{t}, \sim_{f}, \wedge_{f}$ and $\vee_{f}$, as well as the first and fourth components of the quadruple truth values, the proof of Theorem 2.6 becomes that of the following proposition: A sequent in this fragment is FT-valid iff it is provable in the above subsystem.

In Pietz-Rivieccio [4], this logic has been studied as Exactly True Logic and its "Hilbert-style" axiomatization has been given.

Incidentally, if $d e s^{\prime \prime}$ is modified to the set $\{(1,1),(0,1)\}$ or $\{(0,1),(0,0)\}$, then the logic determined by the modified validity coincides with the first degree entailment.

\section{Adding propositional constants}

In this section, the propositional constant $c\langle n, f, t, b\rangle$ such that

$$
v(c\langle n, f, t, b\rangle)=(n, f, t, b), \text { for every valuation } v
$$

is introduced for each quadruple truth value $(n, f, t, b) \in\{1,0\}^{4}$.

Definition 5.1. The system $\mathrm{L}_{b l}^{+}$is obtained from $\mathrm{L}_{b l}$ by supplementing the following axioms, where in (D6), $n^{\prime \prime}=n \wedge n^{\prime}, f^{\prime \prime}=f \wedge f^{\prime}, t^{\prime \prime}=t \vee t^{\prime}$ and $b^{\prime \prime}=b \vee b^{\prime}$ : 
(D1) $\Rightarrow c\langle n, 0,1, b\rangle$

(D2) $c\langle n, 1, t, b\rangle \Rightarrow$ and $c\langle n, f, 0, b\rangle \Rightarrow$

(D3) $\sim_{t} c\langle n, f, t, b\rangle \vee_{t} C \Longleftrightarrow c\langle t, b, n, f\rangle \vee_{t} C$

(D4) $\sim_{f} c\langle n, f, t, b\rangle \vee_{t} C \Longleftrightarrow c\langle f, n, b, t\rangle \vee_{t} C$

(D5) $\sim_{b} c\langle n, f, t, b\rangle \vee_{t} C \Longleftrightarrow c\langle b, t, f, n\rangle \vee_{t} C$

(D6) $c\langle n, f, t, b\rangle \vee_{t} c\left\langle n^{\prime}, f^{\prime}, t^{\prime}, b^{\prime}\right\rangle \Longleftrightarrow c\left\langle n^{\prime \prime}, f^{\prime \prime}, t^{\prime \prime}, b^{\prime \prime}\right\rangle$

It will be evident that all the supplemented axioms are $b l$-valid.

REMARK 5.2. (1) If we don't mind too much dependence on the particular constant $c\langle 0,0,0,0\rangle$ in the axioms, the formulas $C$ occurring in Axioms (D3)-(D5) and $c\left\langle n^{\prime}, f^{\prime}, t^{\prime}, b^{\prime}\right\rangle$ in (D6) can be specified to the constant $c\langle 0,0,0,0\rangle$. More radically, the supplemented axioms can be replaced with the sequents in Lemma 5.3 all together.

(2) Axiom (A7) is superflous in $\mathrm{L}_{b l}^{+}$, since it is obtained from the following axioms by applications of (Cut):

$$
\begin{aligned}
\sim_{b} A & \Rightarrow \sim_{b} A \vee_{t} c\langle 0,0,0,0\rangle \\
\sim_{b} A \vee_{t} c\langle 0,0,0,0\rangle, A & \Rightarrow c\langle 0,0,0,0\rangle \\
c\langle 0,0,0,0\rangle & \Rightarrow
\end{aligned}
$$

LEMMA 5.3. The following sequents are provable.

(1) $\Rightarrow c\langle 0,0,0,0\rangle \vee_{t} c\langle 1,1,1,1\rangle$

(2) $c\langle 0,0,0,0\rangle \Rightarrow \quad$ and $\quad c\langle 1,1,1,1\rangle \Rightarrow$

(3) $\Rightarrow c\langle n, f, 1, b\rangle \vee_{t} c\langle 0,0,0,0\rangle \quad$ and $\quad c\langle n, f, 0, b\rangle \vee_{t} c\langle 0,0,0,0\rangle \Rightarrow$

(4) $\Rightarrow \sim_{t} c\langle 1, f, t, b\rangle \vee_{t} c\langle 0,0,0,0\rangle \quad$ and $\quad \sim_{t} c\langle 0, f, t, b\rangle \vee_{t} c\langle 0,0,0,0\rangle \Rightarrow$

$(5) \Rightarrow \sim_{f} c\langle n, f, t, 1\rangle \vee_{t} c\langle 0,0,0,0\rangle \quad$ and $\quad \sim_{f} c\langle n, f, t, 0\rangle \vee_{t} c\langle 0,0,0,0\rangle \Rightarrow$

(6) $\Rightarrow \sim_{b} c\langle n, 1, t, b\rangle \vee_{t} c\langle 0,0,0,0\rangle \quad$ and $\quad \sim_{b} c\langle n, 0, t, b\rangle \vee_{t} c\langle 0,0,0,0\rangle \Rightarrow$

Proof: All sequents are easily obtained from supplemented axioms by applications of (Cut), and so only a few of them are mentioned. (1) follows from the sequents $\Rightarrow c\langle 0,0,1,1\rangle$ and $c\langle 0,0,1,1\rangle \Rightarrow c\langle 0,0,0,0\rangle \vee_{t}$ $c\langle 1,1,1,1\rangle$, which are ones of Axioms (D1) and (D6) respectively, by (Cut). (2) are two of Axiom (D2). The latter of (4) follows from the following axioms by (Cut)'s: 


$$
\begin{aligned}
\sim_{t} c\langle 0, f, t, b\rangle \vee_{t} c\langle 0,0,0,0\rangle & \Rightarrow c\langle t, b, 0, f\rangle \vee_{t} c\langle 0,0,0,0\rangle \\
c\langle t, b, 0, f\rangle \vee_{t} c\langle 0,0,0,0\rangle & \Rightarrow c\langle 0,0,0, f\rangle \\
c\langle 0,0,0, f\rangle & \Rightarrow
\end{aligned}
$$

Corollary 5.4. Suppose that $\alpha$ forms a complete consistent system.

(1) $c\langle 0,0,0,0\rangle \vee_{t} c\langle 1,1,1,1\rangle \in \alpha$.

(2) $c\langle 0,0,0,0\rangle, c\langle 1,1,1,1\rangle \notin \alpha$.

Proof: Immediate from Lemma 5.3 (1) and (2), respectively.

According to Corollary 5.4, the condition $(*)$ introduced in Section 3 is not the case. So, we let $F(p)$ be $p \vee_{t} c\langle 0,0,0,0\rangle$. Then Lemma 3.3 is valid in this case too. Moreover, Lemma 3.5 and so Lemma 3.6 also hold by Corollary 5.5 below.

Corollary 5.5. Let $\alpha$ be a complete consistent system, and $v^{0}$ the valuation defined in Definition 3.4.

(1) $v_{n}^{0}(c\langle n, f, t, b\rangle)=1$ iff $\sim_{t} c\langle n, f, t, b\rangle \vee_{t} c\langle 0,0,0,0\rangle \in \alpha$.

(2) $v_{f}^{0}(c\langle n, f, t, b\rangle)=1$ iff $\sim_{b} c\langle n, f, t, b\rangle \vee_{t} c\langle 0,0,0,0\rangle \in \alpha$.

(3) $v_{t}^{0}(c\langle n, f, t, b\rangle)=1$ iff $c\langle n, f, t, b\rangle \vee_{t} c\langle 0,0,0,0\rangle \in \alpha$.

(4) $v_{b}^{0}(c\langle n, f, t, b\rangle)=1$ iff $\sim_{f} c\langle n, f, t, b\rangle \vee_{t} c\langle 0,0,0,0\rangle \in \alpha$.

Proof: Immediate from Lemma 5.3 (4), (6), (3) and (5), respectively.

Hence, we have Theorem 2.6 for $\mathrm{L}_{b l}^{+}$, too.

ACKNowledgements. The author would like to thank the anonymous referees for their helpful comments, in which information on Pietz-Rivieccio's paper [4] is included, on the earlier version of this article.

\section{References}

[1] O. Arieli and A. Avron, Reasoning with logical bilattices, Journal of Logic, Language and Information 5 (1996), pp. 25-63.

[2] S. Maehara, A general theory of completeness proofs, Annals of the Japan Association for Philosophy of Science 3:5 (1970), pp. 242-256. 
[3] S. P. Odintsov, On axiomatizing Shramko-Wansing's logic, Studia Logica 91 (2009), pp. 407-428.

[4] A. Pietz and U. Rivieccio, Nothing but the truth, Journal of Philosophical Logic 42 (2013), pp. 125-135.

[5] Y. Shramko and H. Wansing, Some usuful 16-valued logics: How a computer network should think, Journal of Philosophical Logic 34 (2005), pp. 121153.

[6] M. Takano, Gentzenization of trilattice logics, Studia Logica 104 (2016), pp. 917-929.

Professor Emeritus

Niigata University

Niigata 950-2181, Japan

e-mail: takano@emeritus.niigata-u.ac.jp 